\title{
Expressão do complexo de histocompatilidade principal de classe I (MHC I) no sistema nervoso central: plasticidade sináptica e regeneração
}

\author{
Expression of class I major histocompatibility complex (MHC I) in the \\ central nervous system: role in synaptic plasticity and regeneration \\ Expresión del complejo principal de histocompatibilidad de clase I (MHC I) \\ en el sistema nervioso central: plasticidad sináptica y regeneración
}

\author{
Renata Graciele Zanon ${ }^{1}$ \\ Amanda Emirandetti ${ }^{1}$ \\ Gustavo Ferreira Simões ${ }^{2}$ \\ Camila Marques Freria ${ }^{3}$ \\ Sheila Cristina Victório ${ }^{2}$ \\ Luciana Politti Cartarozzi ${ }^{4}$ \\ Roberta Barbizan ${ }^{2}$ \\ Alexandre Leite Rodrigues de Oliveira ${ }^{5}$
}

\section{RESUMO}

Foi demonstrado recentemente que o complexo de histocompatibilidade principal de classe I (MHC I), expresso no sistema nervoso central (SNC), não funciona somente como molécula com papel imunológico, mas também como parte de um mecanismo envolvido na plasticidade sináptica. A expressão de MHC I interfere na intensidade e seletividade da retração de sinapses em contato com neurônios que sofreram lesão e também influencia a reatividade das células gliais próximas a esses neurônios. $A$ intensidade do rearranjo sináptico $e$ resposta glial após lesão, ligadas à expressão de MHC I no SNC, reper-

\begin{abstract}
It has been recently demonstrated that the major histocompatibility complex of class I (MHC I) expressed in the central nervous system (CNS) does not only function as a molecule of the immune system, but also plays a role in the synaptic plasticity. The expression of MHC I influences the intensity and selectivity of elimination of synapses apposed to neurons that were subjected to lesion, besides influencing the reactivity of neighboring glial cells. MHC $I$ expression and the degree of synaptic rearrangement and glial response after injury correlate
\end{abstract}

\section{RESUMEN}

El complejo mayor de histocompatibilidad de clase I (MHC I), expresado en el sistema nervioso central (SNC), no sólo funciona como una molécula con función inmunológica, sino que es crucial para las respuestas del tejido nervioso en casos de lesiones. El MHC I está involucrado con los procesos de plasticidad sináptica y las células gliales en el microambiente de la médula espinal después de realizada axotomía periférica. La expresión de MHC I interfiere con la intensidad y la forma en que se producen la contracción y la eliminación de sinapsis con relación a las neuronas, cuyos axones se han comprometido, y también influye en la reactividad de las

\footnotetext{
Laboratório de Regeneração Nervosa do Departamento de Anatomia, Biologia Celular, Fisiologia e Biofísica da Universidade Estadual de Campinas - UNICAMP Campinas (SP), Brasil.

' Doutor em Biologia Celular e Estrutural do Laboratório de Regeneração Nervosa do Departamento de Anatomia, Biologia Celular, Fisiologia e Biofísica da Universidade Estadual de Campinas - UNICAMP - Campinas (SP), Brasil.

2 Pós-graduando (Doutorado) em Biologia Celular e Estrutural; Laboratório de Regeneração Nervosa do Departamento de Anatomia, Biologia Celular, Fisiologia e Biofísica da Universidade Estadual de Campinas - UNICAMP - Campinas (SP), Brasil.

${ }^{3}$ Pós-graduando (Doutorado) em Clínica Médica; Laboratório de Regeneração Nervosa do Departamento de Anatomia, Biologia Celular, Fisiologia e Biofísica da Universidade Estadual de Campinas - UNICAMP - Campinas (SP), Brasil.

${ }^{4}$ Aluno de Iniciação Científica; Laboratório de Regeneração Nervosa do Departamento de Anatomia, Biologia Celular, Fisiologia e Biofísica da Universidade Estadual de Campinas - UNICAMP - Campinas (SP), Brasil.

${ }^{5}$ Livre-docente; Professor do Laboratório de Regeneração Nervosa do Departamento de Anatomia, Biologia Celular, Fisiologia e Biofísica da Universidade Estadual de Campinas - UNICAMP - Campinas (SP), Brasil.
} 
cute em diferenças na capacidade regenerativa e recuperação funcional em linhagens de camundongos isogênicos. Dessa forma, os novos aspectos sobre a função do MHC I no SNC direcionam futuras pesquisas no sentido de buscar o envolvimento do MHC I em doenças neurológicas e também o desenvolvimento de novas estratégias terapêuticas. with differences in the regenerative potential and functional recovery of isogenic mice strains. In this way, the new aspects regarding MHC I functions in the CNS may guide further studies aiming at searching the involvement of $\mathrm{MCH}$ I in neurologic disorders, as well as the development of new therapeutic strategies. células gliales, cerca de estas neuronas. La intensidad de estos cambios, que responden a la expresión de MHC I en el $S N C$, implica diferencias en la capacidad de regeneración axonal de las células dañadas por axotomía, por lo que el nivel de expresión de las moléculas MHC I se relaciona con el proceso de regeneración de los axones y, en consecuencia, con la recuperación funcional. Por consiguiente, estos nuevos aspectos sobre la función del MHC I en el SNC orientan nuevas investigaciones con miras a entender el papel del MHC I en las enfermedades neurológicas y a desarrollar nuevas estrategias terapéuticas.

\section{DESCRIPTORES: Complejo} mayor de histocompatibilidad; Médula espinal; Axotomía; Plasticidad neuronal; Sinapsis; Axones

\author{
principal de \\ histocompatibilidade; Medula \\ espinal; Axotomia; Plasticidade \\ neuronal; Sinapses; Axônios
}

\author{
KEYWORDS: Major \\ histocompatibility complex; \\ Spinal cord; Axotomy; \\ Neuronal plasticity; Synapses; \\ Axons
}

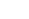



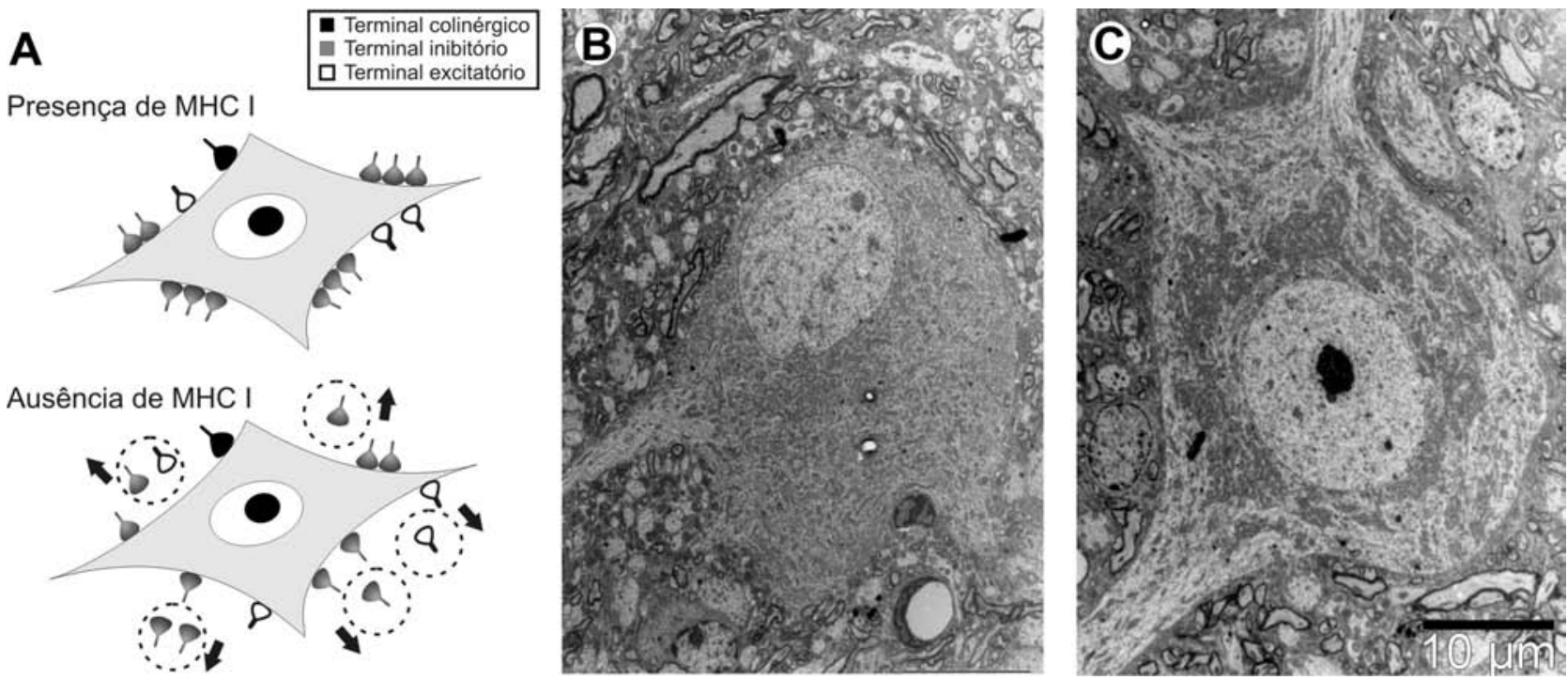

Figura 1

Expressão de MHC I e plasticidade sináptica. (A) Representação esquemática do aumento da perda sináptica resultante de uma lesão axonal na ausência de expressão de $\mathrm{MHC} \mathrm{I;} \mathrm{(B} \mathrm{e} \mathrm{C)} \mathrm{Respectivamente,} \mathrm{um} \mathrm{neurônio} \mathrm{motor} \mathrm{submetido} \mathrm{à} \mathrm{axotomia}$ periférica e um motoneurônio normal, ambos observados sob microscopia eletrônica de transmissão. Escala $=10 \mu \mathrm{m}$.

Também foi observada a correlação da modulação do MHC I no sistema nervoso e a plasticidade sináptica em modelo animal da distrofia muscular de Duchenne (DMD), doença caracterizada pela ausência da proteína distrofina e por ciclos repetitivos de degeneração e regeneração muscular (em camundongos MDX). Observou-se, na medula espinal de camundongos MDX, uma menor cobertura sináptica em relação aos neurônios motores da coluna anterior. Nesse trabalho, os autores sugeriram que essa redução de sinapses fosse decorrente de alterações retrógradas, resultantes da desconexão parcial entre o órgão-alvo e o corpo neuronal durante o período de degeneração da fibra muscular, que ocorre a partir das primeiras semanas de vida nos camundongos $\mathrm{MDX}^{13}$. Tal fato foi correlacionado à menor expressão de MHC I na medula espinal.

\section{Gliose e MHC I}

O processo de plasticidade neuronal está estreitamente relacionado às alterações morfofuncionais que ocorrem nas células gliais, denominado gliose em conjunto. Os astrócitos constituem a maior parte da glia e, por meio de suas amplas funções e da relativa motilidade de suas projeções citoplasmáticas, representam elementos fundamentais na plasticidade sináptica após uma lesão nervosa ${ }^{14}$. Essas projeções respondem com rápidas alterações estruturais ${ }^{15}$, interpondo-se entre a membrana do neurônio motor comprometido por uma lesão e seus terminais sinápticos retraídos $^{14,16}$ (Figura 2).

A microglia, parte do sistema fagocitário mononuclear, reage com hiperplasia e hipertrofia após uma axotomia, e expressa grandes quantidades $\mathrm{MHC} \mathrm{I}^{17}$. Nesse caso, os astrócitos também podem contribuir com a inflamação local e com a expressão de MHC I ${ }^{9,18}$.
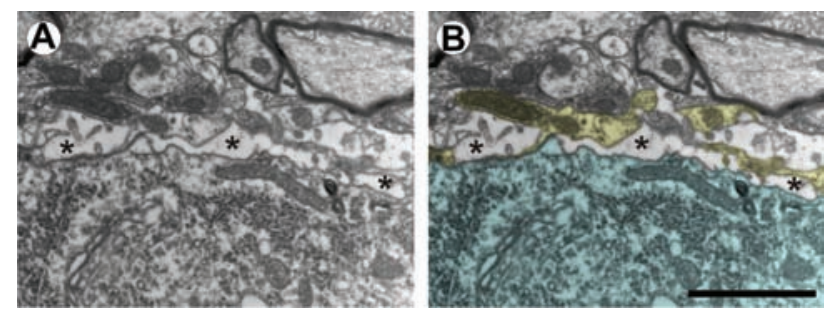

Figura 2

(A) Micrografia eletrônica de transmissão ilustrando o processo de retração sináptica, uma semana após axotomia periférica. Em (B), a imagem anterior foi colorizada para a diferenciação dos elementos pré e pós-sinápticos: terminais pré-sinápticos retraídos (amarelo), membrana pós-sináptica do neurônio motor (azul). Os asteriscos indicam as projeções astrocitárias que ocupam o espaço da retração. Escala $=2 \mu \mathrm{m}$.

A modulação da expressão de MHC I interfere no nível de reatividade dos astrócitos, alterando o processo de plasticidade sináptica ${ }^{14}$. Dessa forma, é estabelecida uma correlação entre MHC I, gliose e plasticidade. Nesse contexto, o MHC I pode ser visto como um mecanismo de comunicação entre neurônios e glia e, de alguma forma contribui, para a coordenação das alterações morfológicas e metabólicas que ocorrem frente a um comprometimento do tecido nervoso.

A resposta aguda a uma lesão tecidual envolve o aumento de citocinas inflamatórias como, por exemplo, o interferon- $\delta$, que é um potente indutor de MHC I. As citocinas são elementos que fazem parte da resposta imune inata e adaptativa, e induzem a expressão da enzima (iNOS) que sintetiza óxido nítrico (NO). Esse é um gás que tem sido relacionado ao processo de apoptose neuronal em diferentes circunstâncias, tanto neuroprotetora ${ }^{19}$ quanto pró-apoptótica ${ }^{20}$. 
Após axotomia periférica, a síntese de NO influencia a expressão de MHC I no microambiente medular, afetando a reatividade dos astrócitos e, consequentemente, a plasticidade das sinapses ${ }^{21}$. Estudos recentes em nosso laboratório mostraram colocalização de MHC I e Iba-1, uma proteína marcadora de microglia. Da mesma forma, em trabalhos anteriores, foi demonstrado que a maior expressão de MHC I está correlacionada a uma intensa reatividade astrocitária ${ }^{22,23}$. Com base nesses achados, sugere-se que as células microgliais, uma vez estimuladas a migrarem ao microambiente de neurônios axotomizados, passam a desencadear uma série de reações que culminam na hipertrofia dos astrócitos. Nesse contexto, pelo menos uma das vias de comunicação celular envolvida seria a ligação entre MHC I e seu receptor, tendo o NO como um dos componentes que possam levar ao aumento da expressão de MHC I pela microglia no SNC (Figura 3).

\section{MHC I e regeneração axonal}

A axotomia de um nervo periférico causa uma resposta tecidual complexa no SNC, afetando tanto o corpo celular quanto o microambiente circunjacente dos neurônios axotomizados. Estruturalmente, observa-se edema do corpo celular, retração de terminações sinápticas, deslocamento do núcleo para a periferia da célula e a dissolução da substância de Nissl. Adicionalmente, alterações metabólicas envolvem o aumento da expressão de proteínas estruturais, como o GAP-43 (growth-associated protein 43), ao passo que a expressão de proteínas relacionadas à transmissão sináptica diminui grandemente ${ }^{24}$. As características de todas essas mudanças permitem a interpretação de um contexto em que os neurônios comprometidos pela lesão passam de um estado funcional de transmissão do impulso nervoso para um modo regenerativo em que a célula direciona seu metabolismo para a recuperação e regeneração ${ }^{25-28}$.

A redução do número de contatos sinápticos está intimamente relacionada à expressão de MHC I no tecido nervoso, que interfere na intensidade do processo de retração e eliminação, bem como na forma como o processo acontece. Assim, os terminais excitatórios são mais afetados, mantendo-se preferencialmente as sinapses inibitórias. Entende-se tal fato como uma estratégia para concentrar o empenho do neurônio em favor do processo regenerativo subsequente ${ }^{12,25-28}$.

Sabha et al..$^{23}$ mostraram a relação entre a capacidade regenerativa e expressão de MHC I em diferentes linhagens de camundongos. Dessa forma, animais da linhagem C57BL6/J, que expressam comparativamente pouca quantidade de MHC I, apresentam baixo potencial regenerativo axonal ao lado de uma menor porcentagem de destacamento sináptico após lesão nervosa. Em contrapartida, animais $\mathrm{A} / \mathrm{J}$, que expressam maior quantidade de $\mathrm{MHC} \mathrm{I}$, mostram uma capacidade regenerativa maior juntamente com uma maior taxa de retração das sinapses.

Contribuindo com essa ideia, experimentos realizados em nosso laboratório mostraram que animais C57BL6/J,
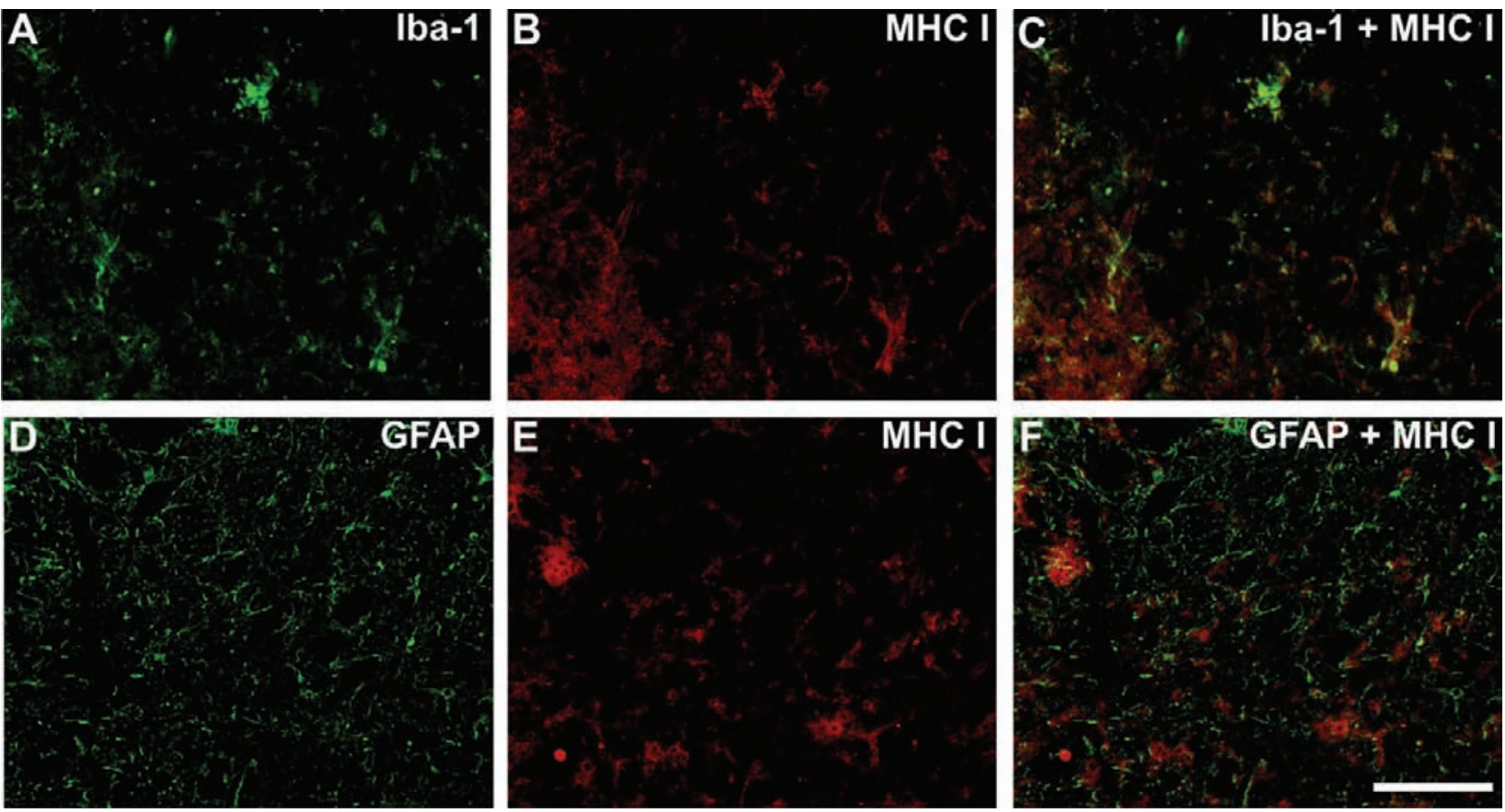

Figura 3

Imunoistoquímica na região do corno anterior da medula espinal de animais submetidos à encefalomietlite autoimune experimental. Em (A) tem-se imunomarcação anti-ibal (marcador microglial), enquanto a figura (D) apresenta imunomarcação anti-GFAP (marcador de astrócitos); (B e E) correspondem à marcação anti-MHC I; (C e F) duplas marcações anti-iba $1 / \mathrm{MHC}$ I e anti-GFAP/MHC I, respectivamente. Nota-se a expressão, especialmente pela microglia, de MHC I (C). Escala = $100 \mu \mathrm{m}$. 
quando tratados com interferon- $\beta$, citocina que eleva a expressão de MHC I após axotomia periférica, apresentam uma aceleração do processo regenerativo axonal e também uma recuperação motora mais rápida em relação aos animais não tratados da mesma linhagem ${ }^{29}$.

\section{MHC I e esclerose múltipla}

De etiopatologia ainda desconhecida, a esclerose múltipla (EM) é considerada, atualmente, uma doença multifatorial, na qual existe a predisposição genética associada a fatores ambientais externos que, uma vez presentes em um mesmo indivíduo, levam a disfunção do sistema imunológico, desenvolvendo ação autolesiva. Esta é dirigida contra a bainha de mielina, presente fundamentalmente na substância branca do SNC, levando ao processo inflamatório e desmielinizante. A reação autoimune é caracterizada principalmente por intenso infiltrado de linfócitos e células mononucleares autorreativas, desmielinização, lesão axonal e perda de oligodendrócitos, resultando numa condução dos impulsos nervosos deficiente, com o aparecimento dos sintomas e sinais clínicos da doenç ${ }^{30}$.

Em estudos clínicos in vivo de pacientes com EM, estão presentes populações de células $T$ ativadas no sangue periférico e no líquido cefalorraquidiano, que reconhecem componentes da mielina como MBP, PLP e $\mathrm{MOG}^{31}$. Esses linfócitos $\mathrm{T}$ são células produtoras de citocinas que modulam a resposta imune. Interessantemente, a produção de citocinas pró-inflamatórias, como o INF-g, INF-a, INF-b e TNF-a, resulta no aumento da expressão de MHC I em neurônios ${ }^{32}$.

Marques et al. ${ }^{33}$ mostraram que, durante a fase aguda da encefalomielite autoimune experimental (EAE), um modelo da EM induzida em animais, há significativo impacto sobre as sinapses dos neurônios motores medulares. A EAE pode ser induzida pela imunização com mielina e seus componentes. Dependendo do animal imunizado, a doença se manifesta de forma aguda monofásica ou crônica, com surtos e remissões. Ratos Lewis imunizados desenvolvem a fase aguda monofásica da EAE e, nesse momento, apresentam perda de $50 \%$ dos contatos sinápticos na coluna anterior da medula espinal com posterior retorno na fase de remissão da doença ${ }^{33}$.

Embasados nesses resultados, Freria et al..$^{34}$ mostraram que a redução da cobertura sináptica no microambiente medular em animais com EAE é acompanhada pela expressão de MHC I juntamente da reatividade exacerbada das células gliais. Em camundongos imunizados, modelo em que a EAE se desenvolve de forma crônica, observase uma correlação da expressão de MHC I com a retração sináptica e melhora dos sintomas clínicos. Dessa forma, na primeira remissão da doença, fase em que as células nervosas medulares ainda exibem a expressão elevada de MHC I, o animal mostra recuperação das coberturas sináptica e clínica compatível com padrões normais. Todavia, na segunda remissão, em que se identifica pequena expressão de MHC I, nota-se que a recuperação dos sinais e sintomas clínicos, bem como o retorno das sinapses, já não alcança níveis de normalidade.

\section{Conclusões e perspectivas}

A expressão da molécula de MHC I contribui efetivamente para o processo de recuperação após uma lesão do sistema nervoso, interferindo na organização de eventos celulares que têm por objetivo a regeneração tecidual e funcional. Hoje em dia, as pesquisas avançam no sentido de desvendar possíveis receptores para o MHC I, bem como sua via de ativação e ação. Até a atualidade, baseados no que se sabe a respeito do sistema imune, a expressão de receptores como TCR e PirB também foi detectada do $\mathrm{SNC}^{35,36}$, porém, animais knockout para esses receptores não exibem os mesmos comprometimentos da plasticidade sináptica durante o desenvolvimento do sistema nervoso, sugerindo que outros receptores estão possivelmente envolvidos. Como um mecanismo de comunicação entre as células nervosas, o MHC I mostra-se uma molécula passível de modulação, permitindo a interferência nas respostas celulares a lesões ou doenças que acometem o sistema nervoso.

\section{REFERÊNCIAS}

1. Babbitt BP, Allen PM, Matsueda G, Haber E, Unanue ER. Binding of immunogenic peptides to ia histocompatibility molecules. Nature. 1985;317(6035):359-61.

2. Parnes JR. Molecular biology and function of cd4 and cd8. Adv Immunol. 1989;44:265-311.

3. Grommé M, Neefjes J. Antigen degradation or presentation by MHC class I molecules via classical and non-classical pathways. Mol Immunol. 2002;39(3-4):181-202.
4. Neumann H. Control of glial immune functions by neurons. Glia. 2001;36:191-9.

5. Williams KA, Hart DNJ, Fabre JW, Morris PJ. Distribution and quantitation of HLA-ABC and DR (Ia) antigens on human kidney and other tissues. Transplantation. 1980;29(4):274-9.

6. Wong G, Bartlett P, Clarklewis I, Battye F, Schrader J. Inducible expression of $\mathrm{H}-2$ and Ia antigens on brain cells. Nature. 1984;310(5979):688-91.
7. Lindå H, Hammarberg H, Cullheim S, Levinovitz A, Khademi M, Olsson $\mathrm{T}$. Expression of MHC class I and $\beta 2$ microglobulin in rat spinal motoneurons; regulatory influences by INF-gamma and axotomy. Exp Neurol. 1998;150:282-95.

8. Tran CT, Wolz P, Egensperger R, Kosel S, Imai Y, Bise K, et al. Differential expression of MHC class II molecules by microglia and neoplastic astroglia: revelance for the escape of astrocytoma cells from immune surveillance. Neuropathol Appl Neurobiol. 1998;24(4):293-301. 
9. Zanon RG, Oliveira AL. MHC I upregulation influences astroglial reaction and synaptic plasticity in the spinal cord after sciatic nerve transection. Exp Neurol. 2006;200(2):521-31.

10.Lindsley MD, Thiemann FL, Rodriguez M. Expression of class I antigen in C57/BL10 and SJl mice following theiler's virus infection. J Neuropathol Exp Neurol. 1988;47:324.

11. Gogate N, Bakhiet M, Kristensson K, Norrby E, Olsson T. Gamma interferon expression and major histocompatibility complex induction during measles and vesicular stomatitis virus infections of the brain. J Neuroimmunol. 1991;31(1):19-26.

12.Oliveira AL, Thams S, Lidman O, Piehl F, Hökfelt T, Kärre KLAS, et al. A role for MHC class I molecules in synaptic plasticity and regeneration of neurons after axotomy. Proc Natl Acad Sci USA. 2004;101(51):17843-8.

13.Simões GF, Oliveira ALR. Alpha motoneurone input changes in dystrophic MDX mice after sciatic nerve transaction. Neuropathol Appl Neurobiol. 2010;36:55-70.

14.Aldskogius H, Liu L, Svensson M. Glial responses to synaptic damage and plasticity. J Neurosci Res. 1999;58(1):33-41.

15.Derouiche A, Frotsher M. Peripheral astrocyte processes: monitoring by selective immunostaining for the actin-binding ERM proteins. Glia. 2001;36(3):330-41.

16.Brännström T, Kellerth J. Changes in synaptology of adult cat spinal $\alpha$-motoneurons after axotomy. Exp Brain Res. 1998;118(1):1-13.

17.Cullheim S, Thams S. The microglial networks of the brain and their role in neuronal network plasticity after lesion. Brain Res Rev. 2007;55(1):89-96.

18.Jarosinski KW, Massa PT. Interferon regulatory factor- 1 is required for interferon-g-induced MHC class I genes in astrocytes. J Neuroimmunol. 2002;122(1-2): 74-84.
19.Keilhoff G, Fansa H, Wolf G. Neuronal NOS deficiency promotes apoptotic cell death of spinal cord neurons after peripheral nerve transaction. Nitric Oxide. 2004;10(2):101-11.

20.He J, Xiao Y, Zhang L. Cocainemediated apoptosis in bovine coronary endothelial cells: role of nitric oxide. J Pharmacol Exp Ther. 2001;298(1):180-7.

21.Emirandetti A. Influência do MHC clásssico (Ia) e não-clássico (Ib) e da óxido nítrico sintase indizível (iNOS) na reação glial e na plasticidade das sinapses após axotomia periférica [tese]. Campinas (SP): Universidade Estadual de Campinas; 2009.

22.Emirandetti A, Zanon RG, Sahba M Jr, de Oliveira AL. Astrocyte reactivity influences the number of presynaptic terminals apposed to spinal motoneurons after axotomy. Brain Res 2006;1095(1): 35-42.

23.Sabha M Jr, Emirandetti A, Cullheim S, Oliveira ALR. MHC I expression and synaptic plasticity in different mice strains after axotomy. Synapse. 2008;62(2):137-48.

24.Moran LB, Graeber MB. The facial nerve axotomy model. Brain Res Rev. 2004;44(2-3):154-78.

25.Barron KD. Comparative observations on the cytologic reactions of central and peripheral nerve cells to axotomy. In: Kao CC, Bunge RP, Reier PJ, editors. Spinal cord reconstruction. New York: Rave Press; 1983. p. 7-39.

26.Lindå H, Piehl F, Dagerlind A, Verge VM, Arvidsson U, Cullheim S, et al. Expression of GAP-43 mRNA in the adult mammalian spinal cord under normal conditions and after different types of lesions, with special reference to motoneurons. Exp Brain Res. 1992;91(2):284-95.

27.Piehl F, Arvidsson U, Johnson H, Cullheim S, Dagerlind A, Ulfhake B, et al. GAP-43, AFGF, CCK and alpha- and beta-CGRP in rat spinal motoneurons subjected to axotomy and/or dorsal root severance. Eur J Neurosci. 1993;5(10):1321-33.
28.Piehl F, Hammarberg H, Hokfelt T, Cullheim S. Regulatory effects of trophic factors on expression and distribution of CGRP and GAP-43 in rat motoneurons. J Neurosci Res. 1998;51(1):1-14.

29.Zanon RG. Influência da modulação da expressão do MHC I sobre a astrogliose reativa e plasticidade sináptica [tese]. Campinas (SP): Universidade Estadual de Campinas; 2009.

30.Moreira MA, Felipe E, Endes MF, Tilbery CP. Esclerose múltipla. Arq Neuropsiquiat. 2000;58(2-b):460-6.

31.Brandão Co, Ruocco Hh, Farias As. Cytokines and intrathecal Igg synthesis in multiple sclerosis patiens during clinical remission. Arq Neuropsiquiatr. 2005;63(4):914-9.

32. Newmann H, Cavalie A, Jenne De, Wekerle H. Induction of Mhc class I genes in neurons. Science. 1995;269(5223):549-52.

33. Marques KB, Santos LM, Oliveira AL. Spinal motoneuron synaptic plasticity during the course of an animal model of multiple sclerosis. Eur J Neurosci. 2006;24(11):3053-62.

34.Freria CM, Zanon RG, Santos LMB, Oliveira ALR. MHC I expression and glial reaction influence spinal motoneuron synaptic plasticity during the course of experimental autoimmune encephalomyelitis. J Comp Neurol. 2010 (accepted).

35. Syken J, Shatz CJ. Expression of T cell receptor beta locus in central nervous system neurons. Proc Natl Acad Sci USA. 2003;100(22):13048-53.

36.Syken J, Grandpre T, Kanold PO, Shatz CJ. PirB restricts oculardominance plasticity in visual cortex. Science. 2006;313(5794):1795-800.

\section{Correspondência}

Alexandre Leite Rodrigues de Oliveira

Departamento de Anatomia, Biologia

Celular, Fisiologia e Biofísica

Instituto de Biologia da Universidade

Estadual de Campinas - CP 6109

CEP 13083-970 - Campinas (SP), Brasil

Fax: (19) 3521-6295

E-mail: alroliv@unicamp.br 